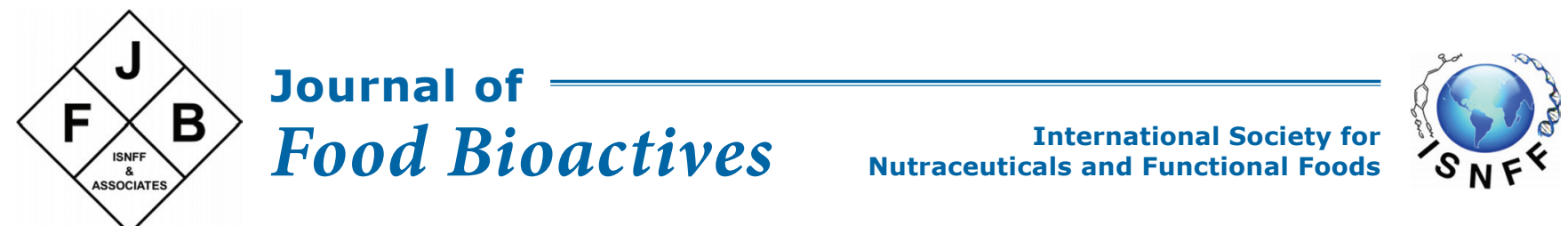

\title{
Qualitative and quantitative changes of nonvolatile compounds and taste profiles in Wuyi rock tea during the manufacturing processes
}

\author{
Xiangyang Guo ${ }^{\mathrm{a}, \mathrm{b}, \mathrm{c}, \mathrm{d}, \mathrm{e}}$, Chi-Tang Ho ${ }^{\mathrm{e}, \mathrm{f}}$, Wilfried Schwabe,g, Zhen Wen ${ }^{\mathrm{a}, \mathrm{b}^{*}}$, Hui Zhu \\ Qiong Liu ${ }^{\mathrm{b}, \mathrm{h}}$, Liang Zhang ${ }^{\mathrm{d}, \mathrm{e}}$ and Xiaochun Wan ${ }^{\mathrm{d}, \mathrm{e}^{*}}$
}

${ }^{a}$ College of Chemistry and Environmental Engineering, Shenzhen University, Shenzhen 518060, China

${ }^{b}$ College of Physics and Optoelectronic Engineering, Shenzhen University, Shenzhen 518060, China

'School of Life Sciences and Food Engineering, Hanshan Normal University, Chaozhou 521041, China

dState Key Laboratory of Tea Plant Biology and Utilization, Anhui Agricultural University, 130 Changjiang Ave W., Hefei, Anhui 230036, China

eInternational Joint Laboratory on Tea Chemistry and Health Effects, Anhui Agricultural University, 130 Changjiang Ave W., Hefei, Anhui 230036, China

fDepartment of Food Science, Rutgers University, New Brunswick, NJ 08901, USA

'Biotechnology of Natural Products, Technische Universität München, Liesel-Beckmann-Str. 1, 85354 Freising, Germany

${ }^{\mathrm{h}}$ College of Life Science and Oceanography, Shenzhen University, Shenzhen 518060, China

*Corresponding author: Xiaochun Wan, State Key Laboratory of Tea Plant Biology and Utilization, International Joint Laboratory on Tea Chemistry and Health Effects, Anhui Agricultural University, 130 Changjiang Ave W., Hefei 230036, China; Tel:+86-551-65786401; E-mail: xcwan@ahau.edu.cn. Zhen Wen, College of Chemistry and Environmental Engineering, Shenzhen University, Shenzhen 518060, China; Tel: +86-755-26557081; E-mail: wenzhen@szu.edu.cn

DOI: $10.31665 /$ JFB. 2021.14270

Received: May 18, 2021; Revised received \& accepted: June 19, 2021

Citation: Guo, X., Ho, C.-T., Schwab, W., Wen, Z., Zhu, H., Liu, Q., Zhang, L., and Wan, X. (2021). Qualitative and quantitative changes of nonvolatile compounds and taste profiles in Wuyi rock tea during the manufacturing processes. J. Food Bioact. 14: 81-93.

\begin{abstract}
Wuyi rock tea (WRT), a type of oolong tea, is famous for 'rock flavor' with characteristic taste and aroma. The qualitative and quantitative changes of the nonvolatile constituents and taste profiles were investigated during the WRT production process by analyzing of polyphenols, caffeine, amino acids, L-theanine and total sugar, coupled with sensory evaluation and determination of dose-over-threshold values. The contents of L-theanine, total sugar, amino acids and some catechins varied considerably except for GA, GCG and GC that increased significantly during the process, while the amount of caffeine was relatively stable. Astringent, bitter, sweet and umami taste properties were predicted. In accordance with the sensory evaluation, there was no umami-like taste in the finished tea that underwent full fire processing, which is essential for WRT to enhance the mellow, heavy taste, and weaken the bitter taste. EGCG and caffeine contributed the most to the astringent, bitter taste of WRT. This study provides a comprehensive profile of the changes in taste characteristics during the WRT manufacturing process.
\end{abstract}

Keywords: Wuyi rock tea; Nonvolatiles; Taste profiles; Dose-over-threshold; Sensory evaluation; Manufacturing process.

\section{Introduction}

Tea (Camellia sinensis) with a pleasant taste and potential health benefits made from the leaves of the tea plant. It is the most popular and consumed beverage besides water around the world (Ho et al., 2015). According to the different tea processing, the tea can be classified into black, white, yellow, green, dark, and oolong tea (Yi et al., 2015). Oolong tea, which is a partially fermented tea is highly appreciated by tea drinkers in Southeast China area and is gaining popularity due to its special flavors (Chen et al., 2010). 
Wuyi rock tea (WRT), one kind of oolong tea, is mainly produced in the Northern Fujian area. It is famous for 'rock flavor' with a characteristic aroma and taste with mellow, heavy and thick attributes, which is produced by a series of manufacturing processes.

Tea manufacturing is the crucial factor that affects tea quality. The production process substantially determines both the properties of the tea flavor and the relevant chemical constituents, and subsequently the nutritional and biological properties of teas (Lin et al., 2015). There are dozens of steps in the conventional WRT manufacturing from fresh tea leaves to the final product, including withering, making (a total of five steps of shaking, i.e. from the first to the fifth shaking), fixing, rolling, roasting twice from the first and second roast, and full fire processing. Tea samples of the individual processing steps have different flavor properties and chemical components, which originate from biochemical reactions or thermal reactions, and form the basis for the flavor generation of the final product tea (Cho et al., 2007). Withering and making are considered to be the vital processes for oolong tea flavor formation. Chemical transformations of plant metabolites and the liberation of flavor compounds occur in these two processes (Lin et al., 2015; Cho et al., 2007; Guo et al., 2019; Ma et al., 2018). During the WRT manufacturing process, enzymes are inactivated in the fixing processing. After this procedure flavor compounds can only be formed non-enzymatically (Kuo et al., 2011). Furthermore, the full fire processing is a special drying procedure, which is a longlasting and highly intensive roasting procedure, and is essential for the production of heterocyclic compounds with nutty or roasted flavor (Guo et al., 2018). A number of early studies focused on the effect of individual process such as withering, making or fixing on the quality of oolong tea, especially on roasting odor formation under high-intensity drying treatment (Hu et al., 2018; Kobayashi et al., 2014; Lin et al., 2016; Sheibani et al., 2016). However, there are few reports of changes in nonvolatile components during the whole WRT processing.

Nonvolatile compounds in the tea leaves have important healthpromoting effects and are precursors of aroma chemicals, and ultiamately influence the sensory characteristics and economic value of the tea (Liu et al., 2018). Some crucial bioactive constituents in tea, such as catechins, caffeine, and amino acids are also important components that determine the taste of the tea infusion (Liu et al., 2018; Mao et al., 2018; Sharma et al., 2018; Yu et al., 2014). Saccharides contribute to the sweet taste and are also important substrates for the production of tea aroma during thermal reactions (Mao et al., 2018; Sharma et al., 2018; Yu et al., 2014; Qu et al., 2019; Jiang et al., 2019). L-theanine, which is the most abundant nonprotein amino acid in teas, shows biological activities and also contributes significantly to the umami taste of tea and is an aroma precursor (Guo et al., 2018; 2019). The nonvolatile components in oolong tea mainly derive from fresh tea leaves. After the leaves are plucked, they undergo various reactions during the tea production process such as aggregation, degradation, oxidation, and isomerization, which result in the formation of flavors and other substances that strongly influence the quality of the tea, especially the taste (Jiang et al., 2019; ÖLmez and Yilmaz, 2009). To the best of our knowledge, the non-volatile compounds in Wuyi rock tea, either in chemical compositions or in taste properties and their changes during production, have been rarely comprehensive analyzed and reported. Knowledge of the composition of nonvolatile substances, their taste profiles and changes throughout the processing chain would assist tea manufacturers either to improve the tea taste or to avoid the generation of undesired flavors.

In the present work, nonvolatile polyphenols, caffeine, L-theanine, total sugar and free amino acids were analyzed in twelve samples at different processing stages from fresh tea leaves to the finished tea product, which was subjected to full fire processing. The aim was to provide the scientific foundation for the processing and quality improvements of Wuyi rock tea.

\section{Experimental}

\subsection{Tea materials}

The preparation of Wuyi rock tea (cultivar "shuixian") with 'rockflavor' was the same to Guo et al. (2018). The oolong tea samples were provided by ManTingFeng Tea Co., Ltd (Wuyi Mountain City, China), and were produced by a tea master with more than 30 years of experience in preparing of tea in 2016 according to the traditional oolong tea processing, including withering, making (a total of five steps, consisted of first shaking, second shaking, third shaking, fourth shaking and fifth shaking), fixing, rolling, roasting twice (first and second roasting) and full fire processing. The detailed parameters of WRT processing were from Guo et al., (2021) and are shown in Table S1. The tea samples immediately frozen in liquid nitrogen and then maintained at $-80{ }^{\circ} \mathrm{C}$ until use. The tea samples corresponding to the process steps were FTL (fresh tea leaves), Wi-OT (withering, oolong tea), 1S-OT (making, first step), 2S-OT (second step), 3S-OT (third step), 4S-OT (fourth step), 5S-OT (fifth step), Fx-OT (fixing, oolong tea), RoOT (rolling, oolong tea), FRt-OT (first roasting), SRt-OT (second roasting) and FF-WRT (full fire, Wuyi rock tea). All tea samples were freeze-dried and were ground into powder with an ultra-mill (Changsha Hongjing Mechanical Equipment Co., Ltd., Changsha, China) until the tea sample could pass through a $350 \mu \mathrm{m}$ sieve.

\subsection{Chemicals}

Deionized water was prepared by a Milli-Q water purification system (Millipore, Billerica, Massachusetts). The authentic standards were all of chromatographic grade. Detailed information of reference standards, solvents and their suppliers are listed in the Table S2.

\subsection{Determination of polyphenols and caffeine}

The preparation of tea infusion and analytical procedure was previously published by Guo et al. (2021). In brief, the extraction of polyphenols and caffeine (CAFF) was conducted two times by adding $5 \mathrm{~mL}$ of $70 \%$ methanol to $0.1 \mathrm{~g}$ of tea powder in a $10 \mathrm{~mL}$ centrifuge tube. The suspension was sonicated with the assistance of KQ-500DE mode ultrasonic (Kunshan Ultrasonic Instruments Co., Ltd., Kunshan, China) for $20 \mathrm{~min}$ at room temperature. The two supernatants were combined and diluted to $10 \mathrm{~mL}$ with $70 \%$ methanol after centrifugation at 3,500 rpm for $10 \mathrm{~min}$, and then filtered through $0.22 \mu \mathrm{m}$ nylon membrane (Baierdi Technology Co. Ltd., Hefei, China). The liquid chromatographic separations were carried out on an Agilent 1260 Infinite II HPLC system using a Kinetex XB-C18 HPLC column $(100 \mathrm{~mm} \times 4.6 \mathrm{~mm}$ i.d., 2.6 $\mu \mathrm{m}$; Phenomenex). Solvent A $(0.2 \%$ acetic acid in water $)$ and $\mathrm{B}$ $(100 \%$ methanol) were as the mobile phase, and the linear elution programing was as follows: $0 \mathrm{~min}, 5 \% \mathrm{~B} ; 2 \mathrm{~min}, 20 \% \mathrm{~B} ; 14 \mathrm{~min}$, $25 \% \mathrm{~B} ; 20$ min, $42 \% \mathrm{~B} ; 22 \mathrm{~min}, 42 \% \mathrm{~B} ; 28 \mathrm{~min}, 100 \% \mathrm{~B} ; 30 \mathrm{~min}$, $5 \% \mathrm{~B}$. The injected volume was $5 \mu \mathrm{L}$ with flow rate of $0.4 \mathrm{~mL} /$ min at $30^{\circ} \mathrm{C}$ and the UV detection wavelength was set at $278 \mathrm{~nm}$. Gallic acid (GA), catechins, and CAFF were identified by compar- 
ing their retention times and UV spectra with those of authentic standards, and were quantitated by external calibration standards. All experiments were run in three replicates.

\subsection{Determination of free amino acids}

The preparation of tea sample was the same to Guo et al. (2021). $0.10 \mathrm{~g}$ of tea powder and $10 \mathrm{~mL}$ of $4 \%$ sulfosalicylic acid was placed in a $10 \mathrm{~mL}$ centrifuge tube and sonicated at $500 \mathrm{~W}$ for 30 min. The supernatant was filtered through a nylon membrane $(0.22$ $\mu \mathrm{m}$, Baierdi Technology Co. Ltd., Hefei, China) after centrifugation at $12,000 \mathrm{rpm}$ for $30 \mathrm{~min}$. The determination of free amino acids was conducted on a L-8900 automatic amino acid analyzer system (Hitachi, Japan). The amino acids were separated by high performance cation-exchange chromatography using a 2622SCPH ion separation column ( $4.6 \mathrm{~mm}$ ID $\times 60 \mathrm{~mm}$ L, Hitachi, Tokyo, Japan, packed with $3 \mu \mathrm{m}$ ion exchange resin) and post-column derivatized by ninhydrin. The temperature of the separation column (Hitachi Ltd.) and reaction column (Hitachi Ltd.) was 57 and 135 ${ }^{\circ} \mathrm{C}$, respectively. The flow rate of pump I (buffer solution, pressure $10.0 \mathrm{MPa}$ ) and II (reaction solution, $1.07 \mathrm{MPa}$ ) was 0.40 and 0.35 $\mathrm{mL} / \mathrm{min}$, respectively. The injected sample volume was $20 \mu \mathrm{L}$ and the detector wavelengths were set at 570 and $440 \mathrm{~nm}$ for signal channel one and two, respectively. The total run time was 32 and $10 \mathrm{~min}$ in signal channel one and two, respectively. The free amino acids were identified by comparing spectra and retention time of the reference amino acids. Quantitation was done via external calibration curves. All experiments were performed in triplicates.

\subsection{Determination of L-theanine}

The analytical method was modified from Guo et al. (2019). The detection was carried out on a Waters Model 2695 HPLC system coupled to a UV detector using a Symmetry Shield RP18 column $(250 \times 4.6 \mathrm{~mm}$ i.d., $5 \mu \mathrm{m}$; Thermo Electron Corp., Waltham, MA, USA). $1.0 \mathrm{~g}$ of tea powder was extracted twice with $100 \mathrm{~mL}$ of boiling distilled water using a KQ-500DE ultrasonicator (500W, Kunshan Ultrasonic Instruments Co., Ltd., Kunshan, China) for 10 min. After filtration, the residue was removed. Subsequently the filtrates were combined and diluted with distilled water to $200 \mathrm{~mL}$ after cooling. Then, the tea infusion was filtered through a $0.45 \mu \mathrm{m}$ polyethersulfone (PES) membrane (Baierdi Technology Co. Ltd., Hefei, China) before subsequent HPLC analysis. The mobile phases consisted of solvent A (water) and B (acetonitrile), and the linear gradient was as follows: $0-10 \mathrm{~min} 100 \% \mathrm{~A}, 10-12$ min linear ramp to $20 \% \mathrm{~A}, 12-20 \mathrm{~min}$ remain $20 \% \mathrm{~A}, 20-22$ min linear ramp to $100 \%$ B, 22-40 min remain $100 \%$ B and the UV detector wavelength was set at $210 \mathrm{~nm}$. The injection volume was $10 \mu \mathrm{L}$ with the flow rate of $1 \mathrm{~mL} / \mathrm{min}$, and the column temperature was $28 \pm 1{ }^{\circ} \mathrm{C}$. L-theanine was identified by comparing absorption spectrum and the retention time with those of the authentic standard. The concentration of L-theanine was calculated from the standard curve. All experiments were run in three replicates.

\subsection{Determination of total sugar}

The tea infusion was prepared by the method of Guo et al. (2018). Briefly, $1.0 \mathrm{~g}$ of tea powder and $80 \mathrm{~mL}$ distilled water with $20 \mathrm{~mL}$ hydrochloric acid was placed in a beaker flask. The mixture was sonicated in a KQ-500DE mode ultrasonic ( $500 \mathrm{~W}$, Kunshan Ultrasonic Instruments Co., Ltd., Kunshan, China) for $15 \mathrm{~min}$. Subsequently, the reaction was carried out in a water bath for $3 \mathrm{~h}$ at $100{ }^{\circ} \mathrm{C}$. This brew was designated as the initial tea infusion. $0.1 \mathrm{~mL}$ of the initial tea infusion was added to a test tube $(10 \mathrm{~mL})$ with glass stopper and diluted with distilled water to $2 \mathrm{~mL}$, and $1.0 \mathrm{~mL}$ of $6 \%$ phenol (w/w, in water) and $5 \mathrm{~mL}$ of concentrated sulfuric acid were added rapidly, the tubes were allowed to stand for $5 \mathrm{~min}$, shaken and placed for 15 min at $100{ }^{\circ} \mathrm{C}$ using a water bath, and then cooled in ice-bath before readings were taken at $490 \mathrm{~nm}$. Blanks were prepared by substituting distilled water for the tea infusion. The amount of total sugar was determined using a standard curve previously generated with for Dglucose. All experiments were run in three replicates.

\subsection{Sensory evaluation}

The method of sensory evaluation on oolong tea was slightly modified from Liu et al. (2018). In brief, $5.0 \mathrm{~g}$ of tea samples from SRt-OT and FF-WRT, respectively, were carried out in a tea cup brewed with $110 \mathrm{~mL}$ of boiling water with the lid put on for 5 min. Then the tea infusion was subjected to a sensory test. All the tea samples were coded using three-digit numbers. After brewing, the samples were randomly offered to panelists to evaluate the tea taste. Panelists recorded the taste descriptors as well as the intensity values of the samples. The six taste attributes, namely mellow, bitter, astringent, sweet, heavy and thick were identified to describe the oolong tea taste by the panelists. The intensities of the taste attributes were scored using a scale from 0 to 10 , where $0=$ none or not perceptible intensity, $3=$ weak intensity, $5=$ moderate intensity, $7=$ high intensity, and $10=$ extremely high intensity. Each sample was evaluated three times by each panelist on different days, and the taste intensity was expressed as the average from all the panelists.

The taste of tea samples was evaluated by the trained panelists, which included six females and four males from 24 to 45 years old. All assessors had more than 5 years of experience in describing the taste attributes of teas. Panelists were trained by a series of important taste compounds including glucose as sweet taste, citric acid as sour taste, caffeine as bitter taste, salt $(\mathrm{NaCl})$ as salty taste, EGCG as astringent taste, and sodium glutamate as umami taste.

\subsection{Statistical analysis}

All the determination were performed in triplicates. The data were analyzed with one-way analysis of variance (ANOVA) and Duncan's multiple-range tests by use of SPSS Statistics 22 (SPSS Inc., Chicago, IL, USA) to determine the statistical difference ( $p$-value $<0.05)$.

\section{Results and discussion}

\subsection{Changes in nonvolatile compounds during the WRT pro- cessing}

\subsubsection{Gallic acid, catechins and caffeine}

The concentrations of eight polyphenols including seven catechins such as EGCG, EGC, ECG, EGC, EC, GC and C as well as one phenolic acid GA were quantitated during the WRT manufacturing processes. They were grouped into two categories according to their trends in their concentration changes. The contents of GA, GC and GCG increased in the late stages of the process and the 

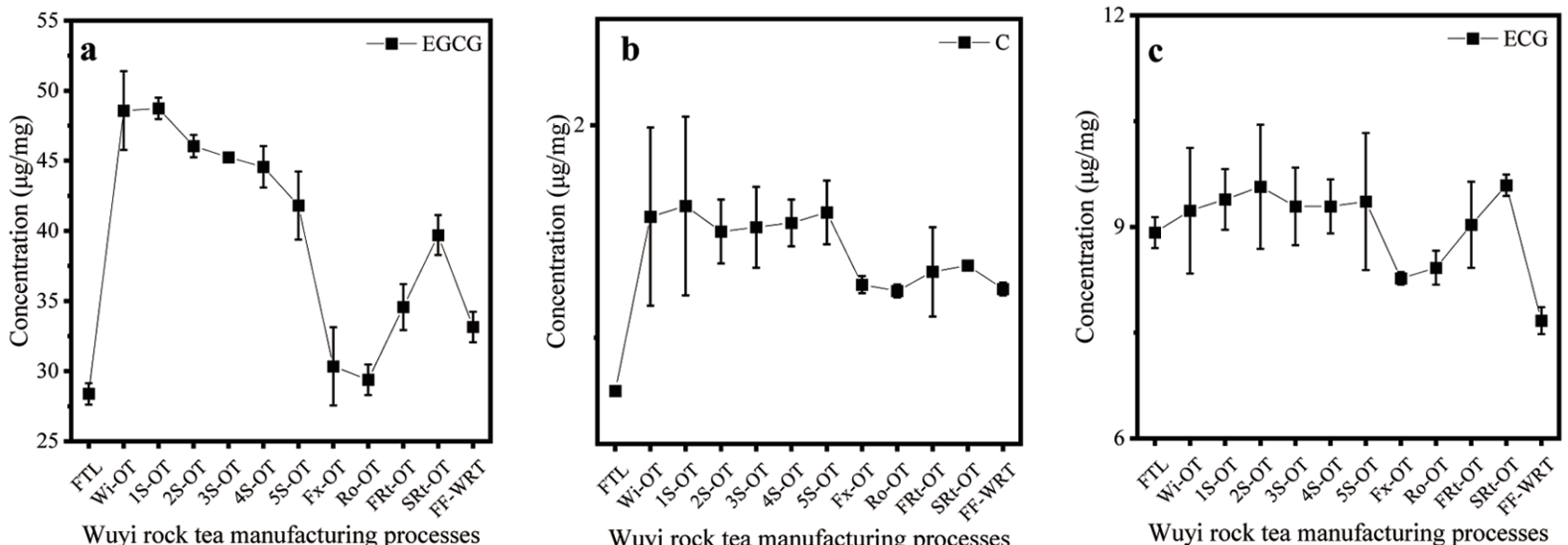

Wuyi rock tea manufacturing processes

Wuyi rock tea manufacturing processes
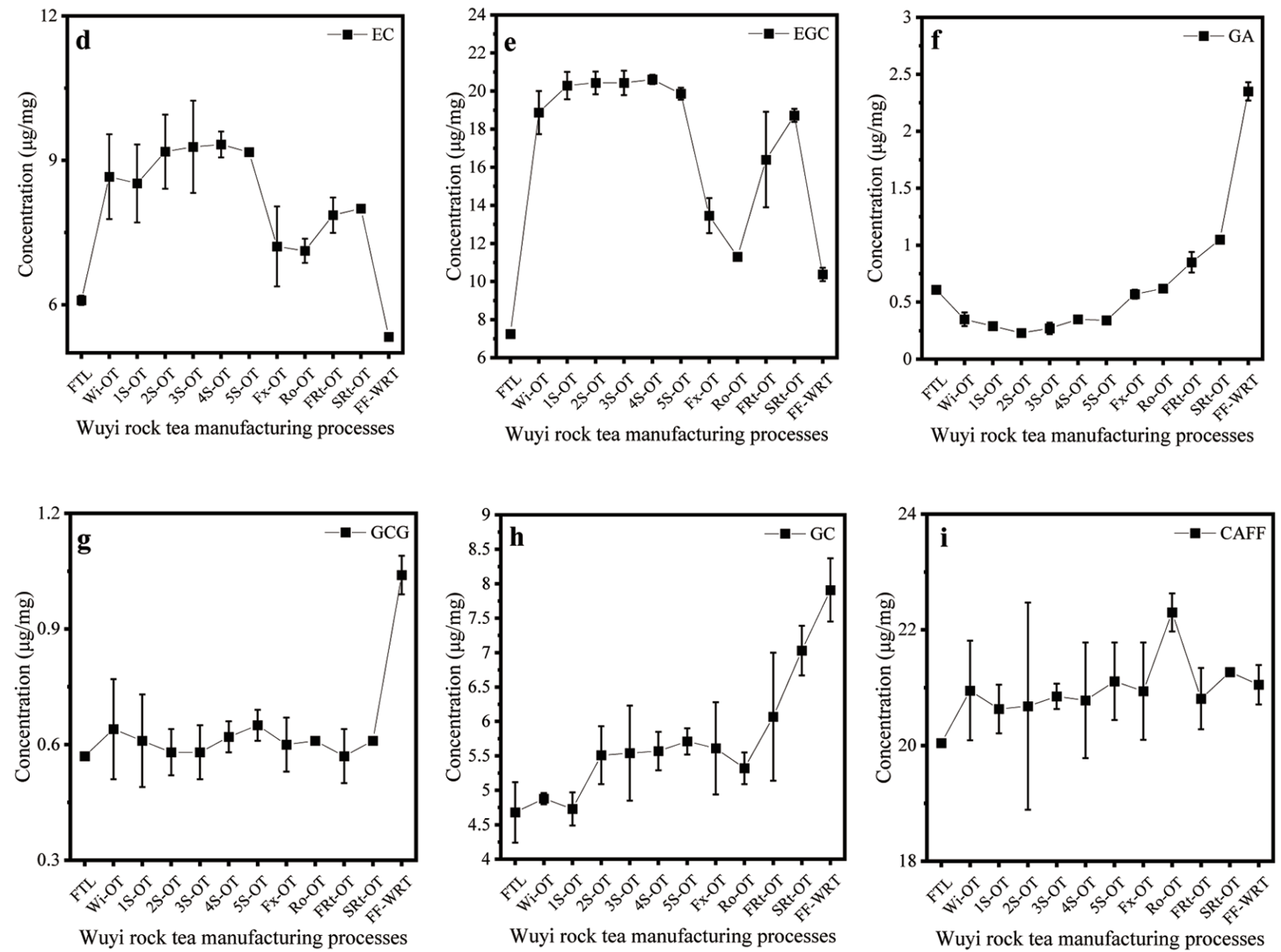

Figure 1. The contents of polyphenols and caffeine during Wuyi rock tea manufacturing processes. (a) EGCG; (b) C; (c) ECG; (d) EC; (e) EGC; (f) GA; (g) GCG; (h) GC; (i) CAFF.

others showed mixed trends (Figure 1). GA and GC contents gradually increased up to of 2.35 and $7.91 \mu \mathrm{g} / \mathrm{mg}$, respectively, until the final stage of FF-WRT, while the GCG level remained stable but rose sharply in FF-WRT to $1.04 \mu \mathrm{g} / \mathrm{mg}$, which was significantly higher than the levels found in other stages. As for other compounds, a similarity was that the content in Wi-OT was higher than 


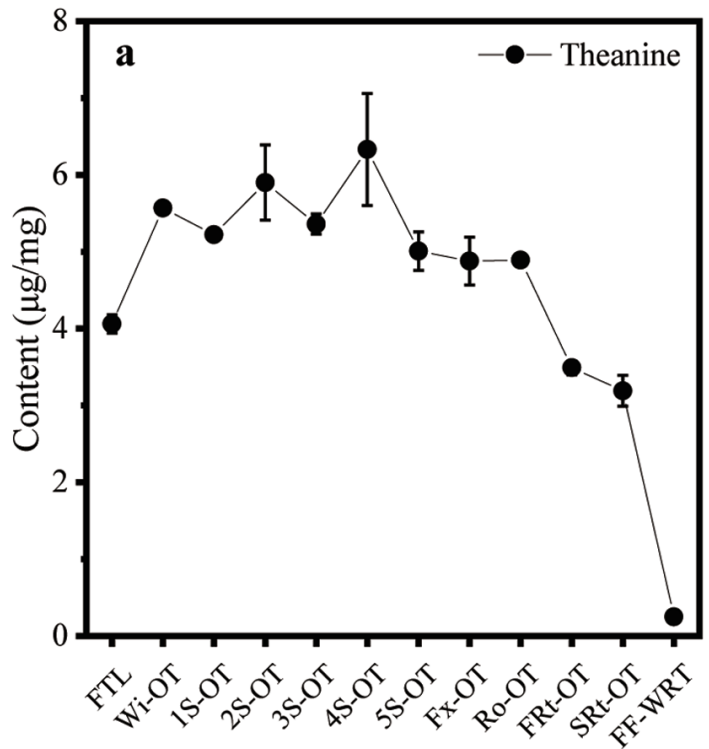

Wuyi rock tea manufacturing processes

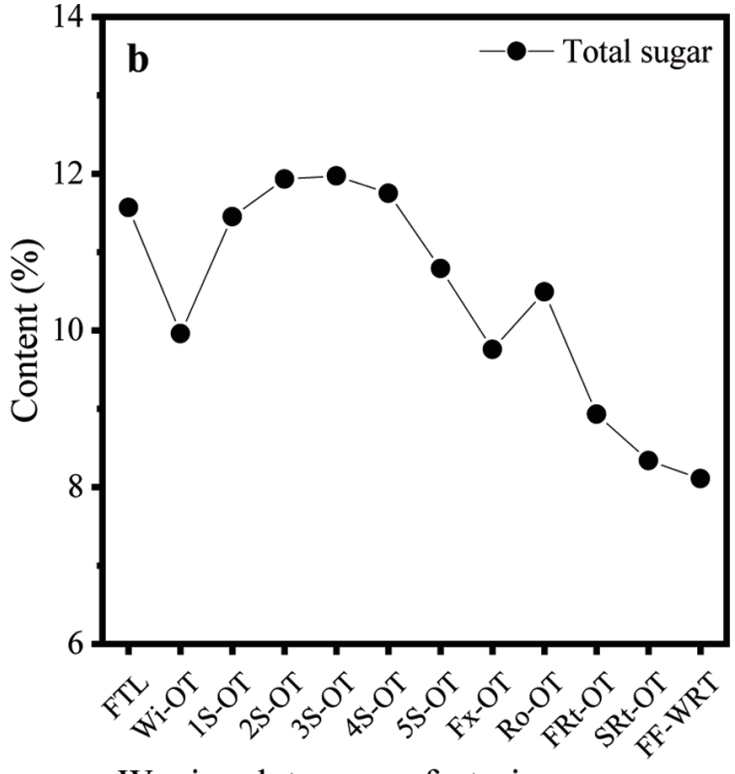

Wuyi rock tea manufacturing processes

Figure 2. The contents of L-theanine and total sugar during Wuyi rock tea manufacturing processes. (a) L-Theanine. (b) Total sugar.

that in FTL and then the content was relatively stable during the making stages. The concentrations were significantly reduced after the fixing (Fx-OT) and rolling (Ro-OT) treatment, increased during the roasting processing (FRt-OT and SRt-OT), but there was a significant reduction after full firing processing (FF-WRT). For EC and ECG the lowest amount was even determined in FF-WRT. In contrary to the polyphenol content, the caffeine content during tea processing was relative stable in the range of $20.04-22.30 \mu \mathrm{g} /$ $\mathrm{mg}$, in line with the findings in other teas (Mao et al., 2018).

Generally, catechins and EGCG in particular are regarded as the most biologically active ingredients in tea, and they are considered the major compounds imparting bitter and astringent taste, and affecting the color of tea infusion (Wang et al., 2000). The processes involved in the manufacturing of tea play a vital role in the dynamic content changes of catechins, which are caused by the hydrolysis of ester-catechins (EGCG, GCG and ECG), enzymatic oxidations, polymerizations and non-enzymatic reactions of catechins (Seto et al., 1997). Probably for these reasons, the amounts of EC and ECG in the final product tea were significantly lower than in FTL, while the contents of other polyphenols in tea were significantly higher than in FTL. The level of EGCG was significantly higher than the levels of the other polyphenols in all tea samples suggesting that EGCG was one of the major contributors for astringency taste in WRT. It should be noted that some catechins showed a significant increase in quantity, such as GC and GCG, or a significant reduction in content, such as EGCG, EGC, ECG and EC, from the SRt-OT to the FF-WRT stage. This observation is mainly due to the epimerization of EGCG, EGC, ECG and EC at the C-2 position of the flavan-3-ols under thermal treatment (Seto et al., 1997). The GA content significantly raised during the WRT manufacturing process. The increase could be due to the degradation of ester catechins to GA and non-ester catechins (EGC, EC and C) by enzymatic conversion, microorganisms, and thermal reaction (ÖLmez and Yilmaz, 2009). In addition to catechins, caffeine also can give a bitter taste to WRT and its bitterness intensity is much higher than that of catechins, therefore the bitterness taste could be dominated by the caffeine content (Yu et al., 2014).

\subsubsection{L-Theanine}

L-Theanine, which is known for its potential health benefits and affects the flavor and quality of tea (Sharma et al., 2018). The amount of L-theanine during WRT manufacturing is illustrated in Figure 2a (the data of L-theanine in FF-WRT, SRt-OT and FTL was adapted from Guo et al. (2018)). There was a significant difference in L-theanine concentration among these samples, ranging from 0.25 to $6.33 \mathrm{mg} / \mathrm{g}$. The lowest content was measured in FFWRT and the highest in 4S-OT.

From withering to making, there was a significant increase in L-theanine content, which could be explained by the hydrolysis of proteins in tea, catalyzed by plant or microbial enzymes (Deng et al., 2009). Subsequently, the L-theanine content decreased simultaneously with the loss of tea juice and moisture after the fixing and rolling step and then further decreased to the lowest amount in the finished product tea. The significant reduction of L-theanine during the heat treatment confirmed that it is thermally degraded to volatiles, which contribute to the aroma of tea (Guo et al., 2018). It is generally known that L-theanine mainly influences the taste of tea and it is thought to be the major source of umami taste with a low threshold value $(3.44 \mu \mathrm{mol} / \mathrm{L})$ in tea, and ultimately affect the property of tea taste (Sharma et al., 2018).

\subsubsection{Free amino acids in addition to theanine}

Twenty amino acids, including eight essential amino acids were quantitated during the WRT processing (Figure 3). Hydroxyproline (Hyp) was not detected in all tea samples. Some amino acids, such as glutamic acid, glutamine, methionine, tryptophan, histidine, arginine, and proline (Glu, Gln, Met, Trp, His, Arg and Pro, respectively) were not detected in the final product tea, Met, His and Arg were not found in FTL, but were identified during the process and disappeared again after the roasting procedure. Most amino acids were detected in trace amounts not exceeding $1 \mathrm{mg} / \mathrm{g}$ except for aspartic acid (Asp), Glu, Gln, which showed levels higher than 1 


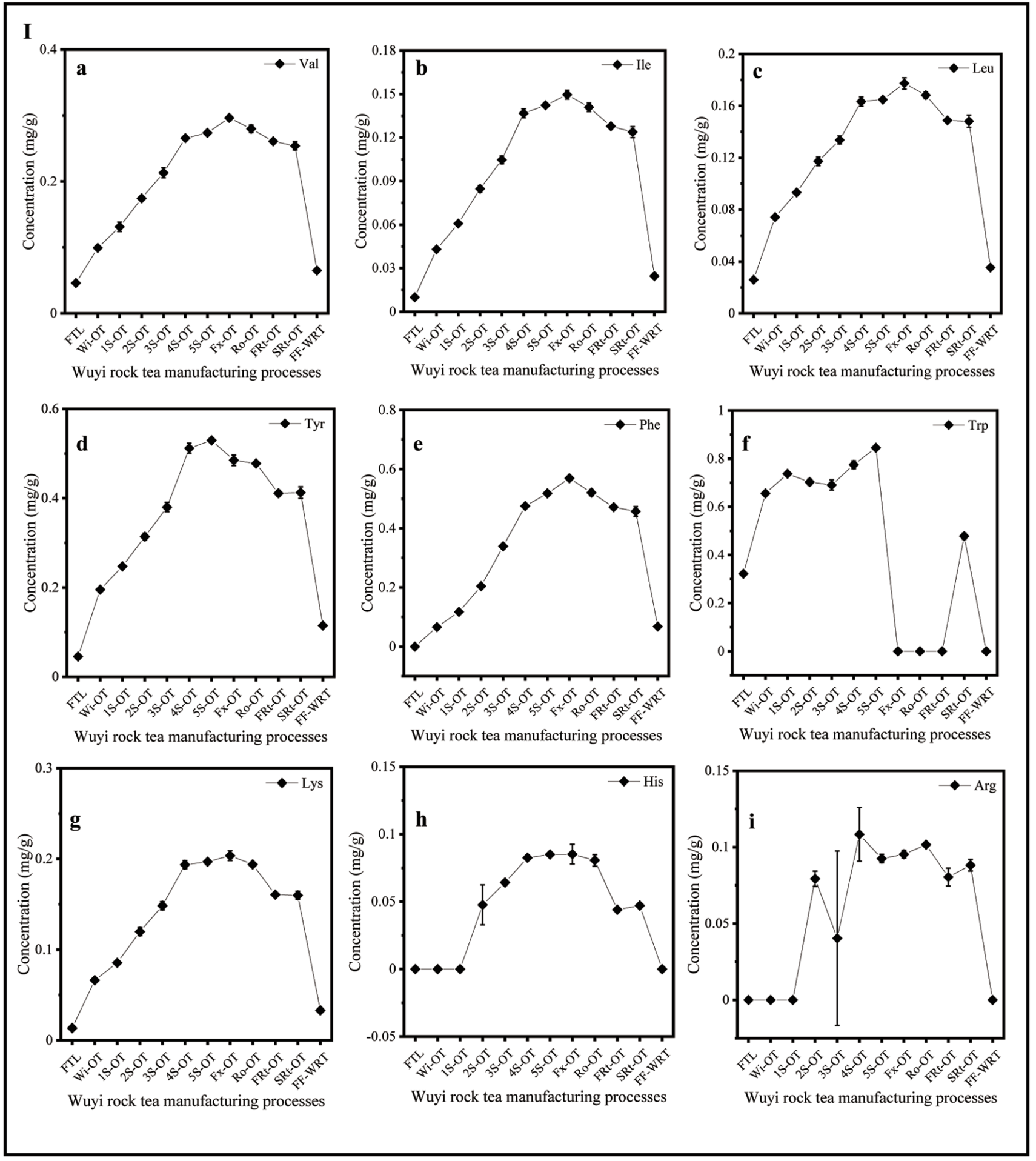

Figure 3. The concentrations of free amino acids during Wuyi rock tea manufacturing processes. (I) Bitter-tasting amino acids, (a) Val; (b) lle; (c) Leu; (d) Tyr; (e) Phe; (f) Trp; (g) Lys; (h) His; (i) Arg. (II) Sweet-tasting amino acids, (j) Thr; (k) Ser; (I) Gln; (m) Gly; (n) Ala; (o) Met; (p) Pro. (III) Umami-tasting amino acids, (q) Asp; (r) Glu. (IV) Other amino acid, (s) Cys. 


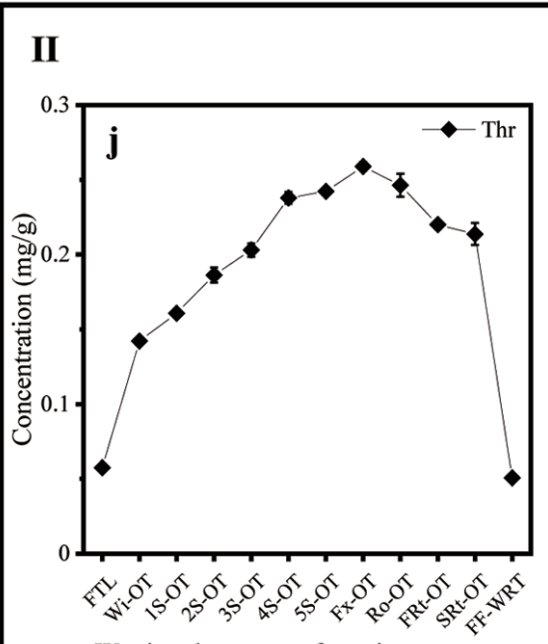

Wuyi rock tea manufacturing processes

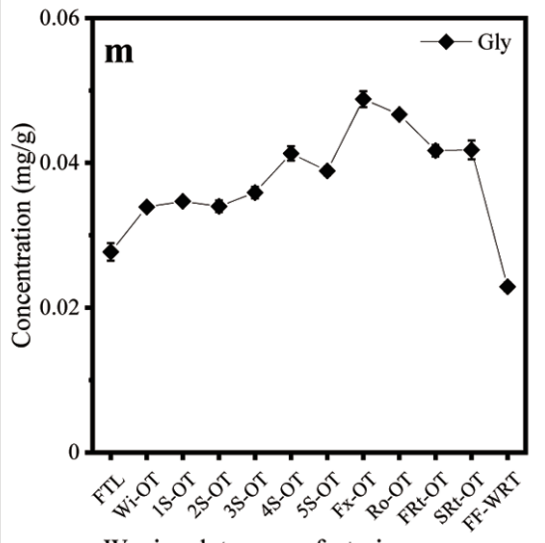

Wuyi rock tea manufacturing processes

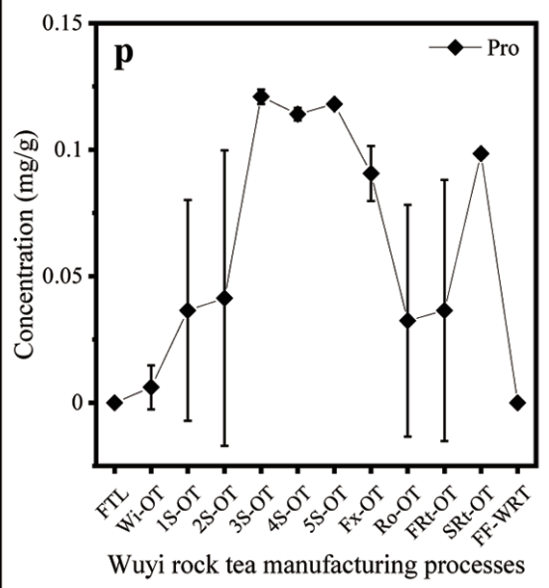

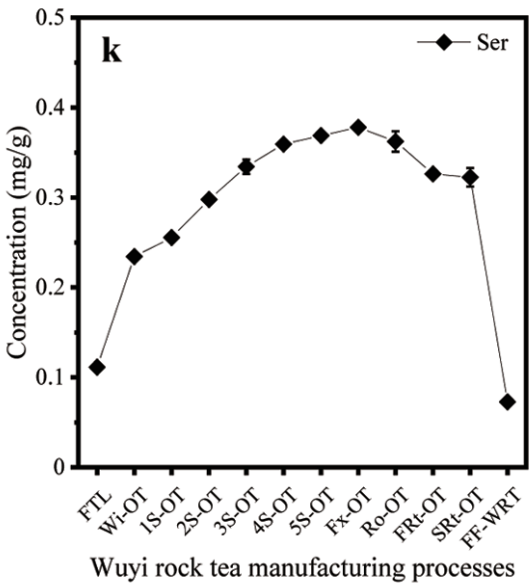
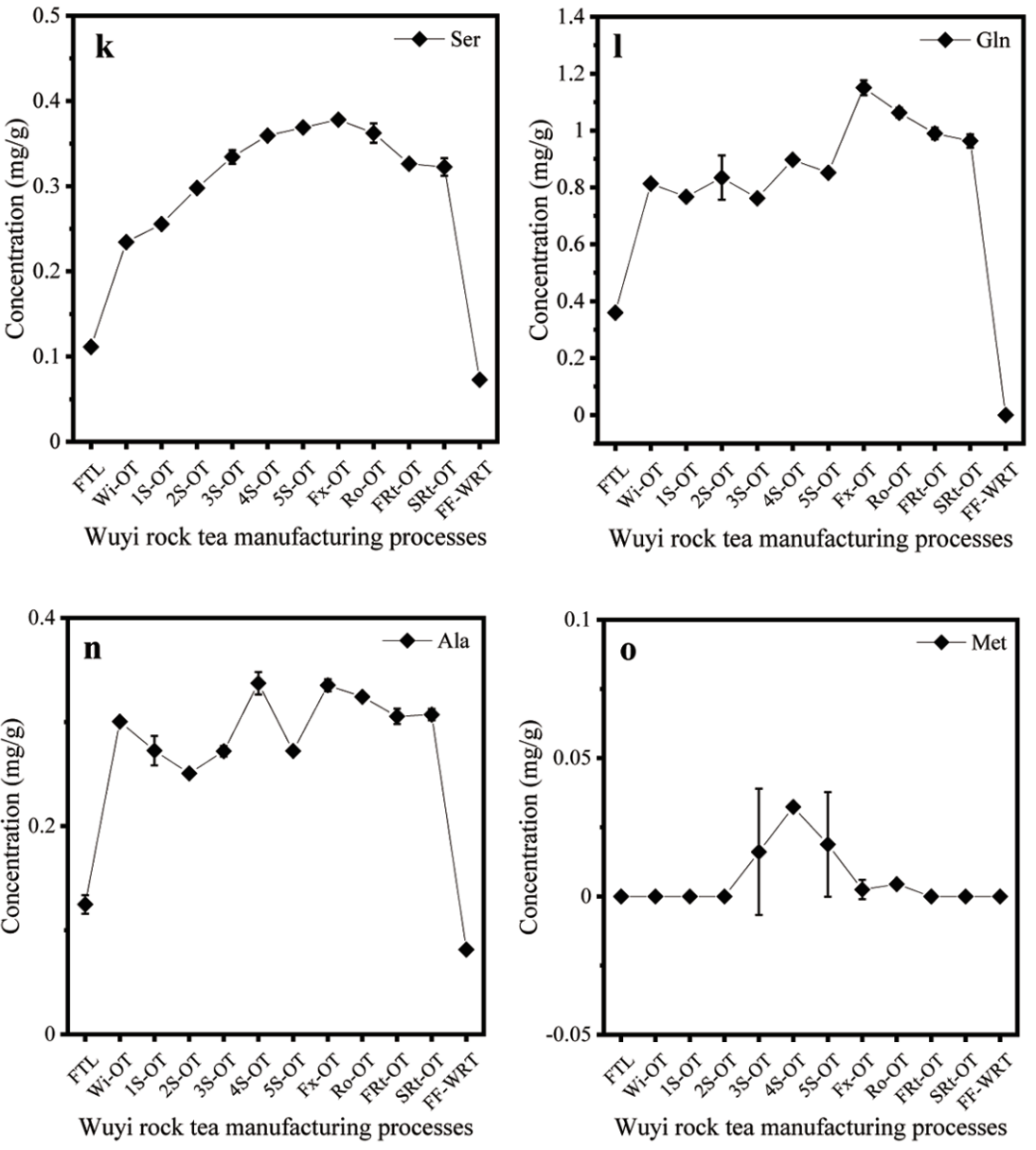

Figure 3. (continued) 


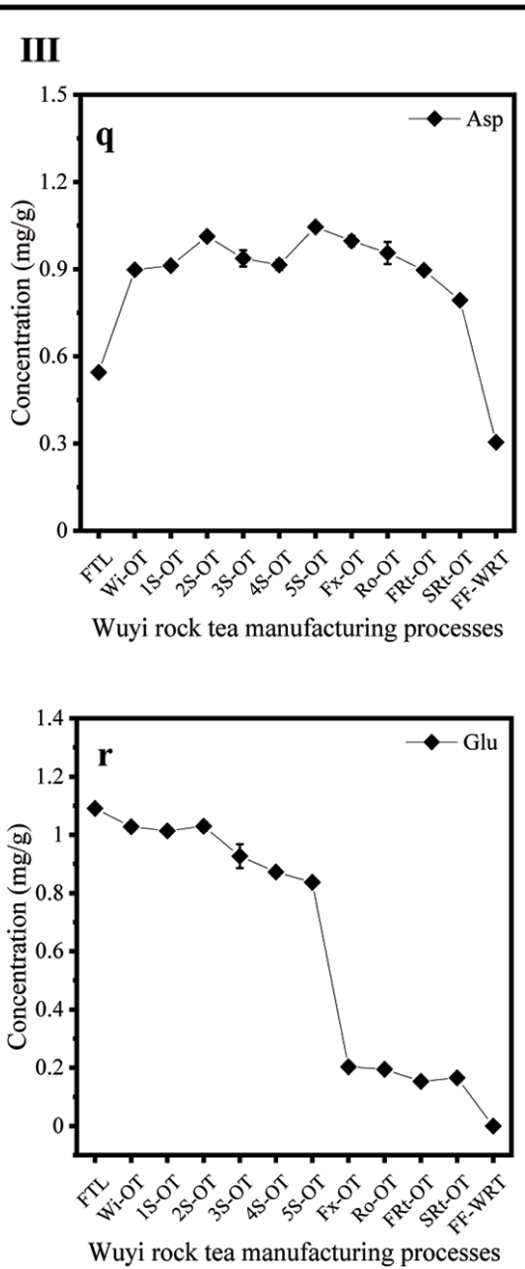

Figure 3. (continued)

$\mathrm{mg} / \mathrm{g}$ in some tea samples. The contents of individual amino acid differed significantly during different stages of the WRT process.

In tea processing, the changes in the amino acid concentration are related to the hydrolysis of proteins, and the oxidation, decarboxylation and thermal reaction of amino acids (Qu et al., 2019; Kausar et al., 2013), subsequently leading to the increase or decrease in amino acid amount. The contents of most amino acids increased due to the hydrolysis and thermal degradation of proteins during withering and fixing, especially during the making phase, and then decreased probably because the amino acids were degraded by thermal reactions after the fixing stage, in which enzyme activities were inactivated (Figure 2). Amino acids are also substrates and intermediates of important biochemical reactions. The variations in the levels of alanine (Ala) and Glu, could be related to the changes of theanine and ethylamine (Deng et al., 2009). In addition, most amino acids, such as phenylalanine (Phe), glycine (Gly), valine (Val), Ala, and Met are important aroma precursors. They react in the Maillard reaction with sugars to liberate volatile compounds with pyrrole, pyrazine, pyran or furan structures. Amino acids also can be transformed to alcohols, phenols or indole (Shahidi et al., 1997). The amino acids Glu, Gln, Met, Trp, His, Arg and Pro were not detected in heat-treated tea samples, suggesting that they were thermally degraded to volatile components.

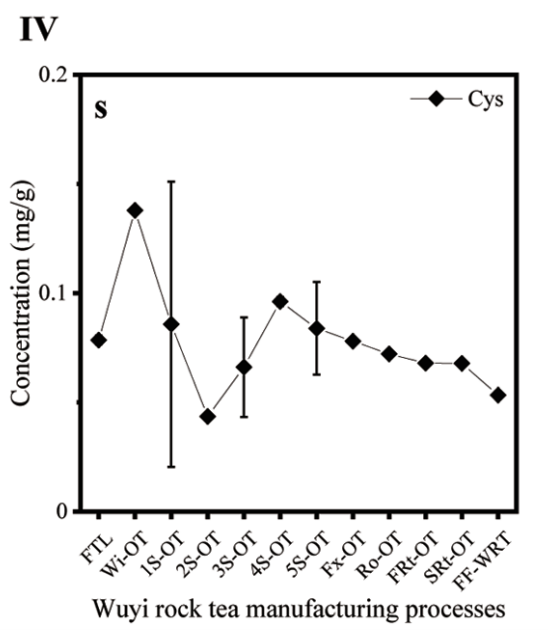

In addition to their contribution to the generation of tea aroma, they are also accountable for the tea taste (Zhang et al., 2019). The amino acids were grouped into three categories, umami, sweet and bitter according to their different taste properties (Mao et al., 2018). The latter two categories contained more amino acids, 8 and 9 , respectively, but the umami group had the highest quantity (Figure 4). Glutamic acid (Glu), which is the major contributor for umami taste together with Asp, was the most abundant amino acid and the only one whose content exceeded $1 \mathrm{mg} / \mathrm{g}$ in fresh tea leaves of WRT, and then gradually decreased during the processing. In contrast to the changes of the Glu concentration, the content of Gly with sweet taste, and His, Phe and Val with bitter taste showed a similar pattern. Their concentrations peaked in the FxOT stage and then decreased significantly. The FF-WRT showed lower amounts of all amino acids than FTL, except for lysine (Lys), tyrosine (Tyr), leucine (Leu), isoleucine (Ile), Val, and Phe.

\subsubsection{Total sugar}

The amount of total sugar was analyzed in tea samples during the WRT processing (Figure 2b, the data of total sugar in FF-WRT, SRt-OT and FTL was adapted from Guo et al. (2018)). Similar to 


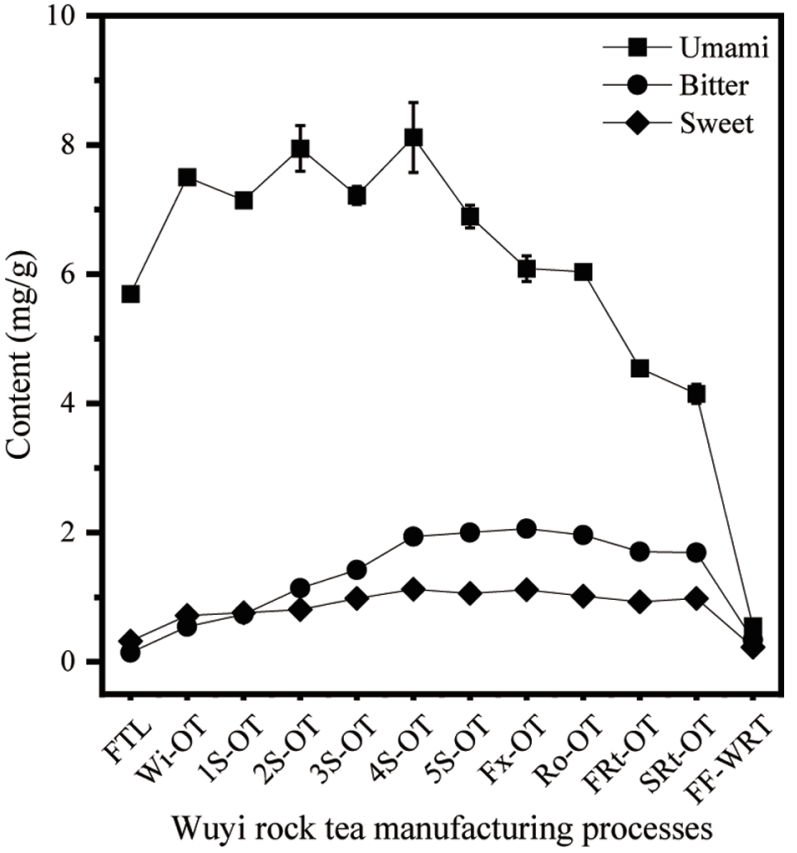

Figure 4. The content of amino acid category during Wuyi rock tea manufacturing processes.

L-theanine, there were significant differences in total sugar content among the samples during processes, peaking during the making phase and the lowest amount in the final product. In contrast to L-theanine, the percentage decrease of total sugar in FF-WRT compared to FTL was $29.90 \%$, while the value for L-theanine was $93.84 \%$. Sugar is the major tastant responsible for sweet taste in tea, and is an important substrate in the Maillard reaction, which contributes to the formation of the tea aroma during tea production (Guo et al., 2018). The lower quantities in tea samples compared to the levels in fresh tea leaves are due to degradation by the Maillard reaction. The monosaccharide composition in tea is diverse and the individual sugars show different reactivities (Bertuzzi et al., 2020). More information is needed to determine precisely the contribution of monosaccharides to the formation of tea flavor. However, the total sugar content may to some extend reflect the effects on the formation of the tea flavor.

\subsection{Taste profiles during the WRT processing}

The taste profile of an individual chemical compound depends on its concentration and taste threshold. The dose-over-threshold (Dot) factors of taste compounds were applied to analyze the taste active compounds in tea (Scharbert and Hofmann, 2005). The Dot factor of the taste-provoking compound was obtained from their concentration by their taste threshold, was used to evaluate the contribution of individual taste component to the taste of tea. Generally, compounds having Dot value of higher than one are assumed to have a positive contribution to the taste of the food. The taste compounds were classified into four groups according to the taste descriptors, including astringent, bitter, and sweet as well as umami-tasting compounds. Detailed information on the taste compounds found in WRT, their taste qualities, thresholds, taste types and Dot values are listed in Table 1.

Table 1 shows that the Dot values of catechins, which have an astringent taste, were all above one during the WRT manufacturing process. Three astringent-tasting compounds, EGCG, EGC and ECG, exhibited Dot factors greater than 60 in the finished product tea, and greater than 100 during the WRT manufacturing process suggesting that they can contribute significantly to the astringent taste of tea. In particular the contribution of EGCG was the highest with Dot factors exceeding 300. Caffeine was the only compound with a Dot factor greater than one in the group of compounds imparting bitter taste. Due to the high Dot value of 216.8 in FF-WRT it has a positive contribution to the bitter taste of WRT. Other bittertasting compounds with Dot factors of less than one have by definition a minor or no effect on the taste of WRT. Similar to group II (bitter-tasting compounds), the sweet-tasting amino acids do not have a positive effect on taste of tea due to Dot factors of less than one except for total sugar. Assuming that the total sugar consists only of glucose, a Dot factor greater than 1 (5.00 in FF-WRT) was calculated in all tea samples, so the sugars were the main contributors for the sweet taste of WRT. Compounds imparting umami-like taste were L-theanine, Asp, and Glu with Dot factors lower than one in FF-WRT. These compounds have little impact on the umami taste of WRT, although the Dot factor was above one in tea samples before full fire processing. The concentrations of umami-like taste compounds decreased significantly after fixing, and sharply decreased due to full fire processing. This indicates that especially heat treatment and high intensity drying had a significant influence on the umami taste of WRT.

Descriptors are used for describing the taste of a compound. The taste compounds that have similar taste properties were grouped together, allowing a taste spectrum with several taste types to be formed to create a taste profile of WRT (Liu et al., 2018; Scharbert and Hofmann, 2005). Four taste types were defined for tea including astringent, bitter, sweet, and umami-like (Table 1). Accordingly, to further understand the variations of the taste compounds during the WRT processing, taste profile webs were established for the non-volatile components for twelve tea samples (Figure 5a). The figure coordinates represent the sum of the Dot factors of the taste compounds of the same taste quality with natural logarithms computation. The primary characteristic taste properties of FTL were a strong astringent taste, moderate bitter taste, weak sweet and umami-like taste (Figure 5a). The intensity of the astringent and bitter taste changed little during the manufacturing process, because of the chemical stability of EGCG and caffeine, and there was no significant variation in the intensity of the sweet taste. However, the tea samples obtained after thermal treatment showed a reduced intensity of the umami-like taste, in particular the FF-WRT sample.

The taste spectrum of the tea infusion during the production process could be determined by calculating the Dot factors. However, since the taste of tea also depends on the interactions of the compounds, the taste profile must be assessed from a sensorial point of view.

\subsection{Sensory evaluation}

Sensory evaluation is an important method for comprehensive assessment of tea taste, and it can clearly distinguish the influence of tea processing on the taste of tea (Nie et al., 2019). Therefore, tea samples of SRt-OT and FF-WRT taken before and after full firing processing were selected for sensory evaluation, in order to analyze the effect of full fire processing on the formation of WRT flavor. The detailed scores on intensity of taste attributes were shown in Table S3. There were significant differences in the taste assessments described for SRt-OT with a strong mellow, sweet, moderately astringent, bitter, thick and heavy taste whereas FF-WRT had 


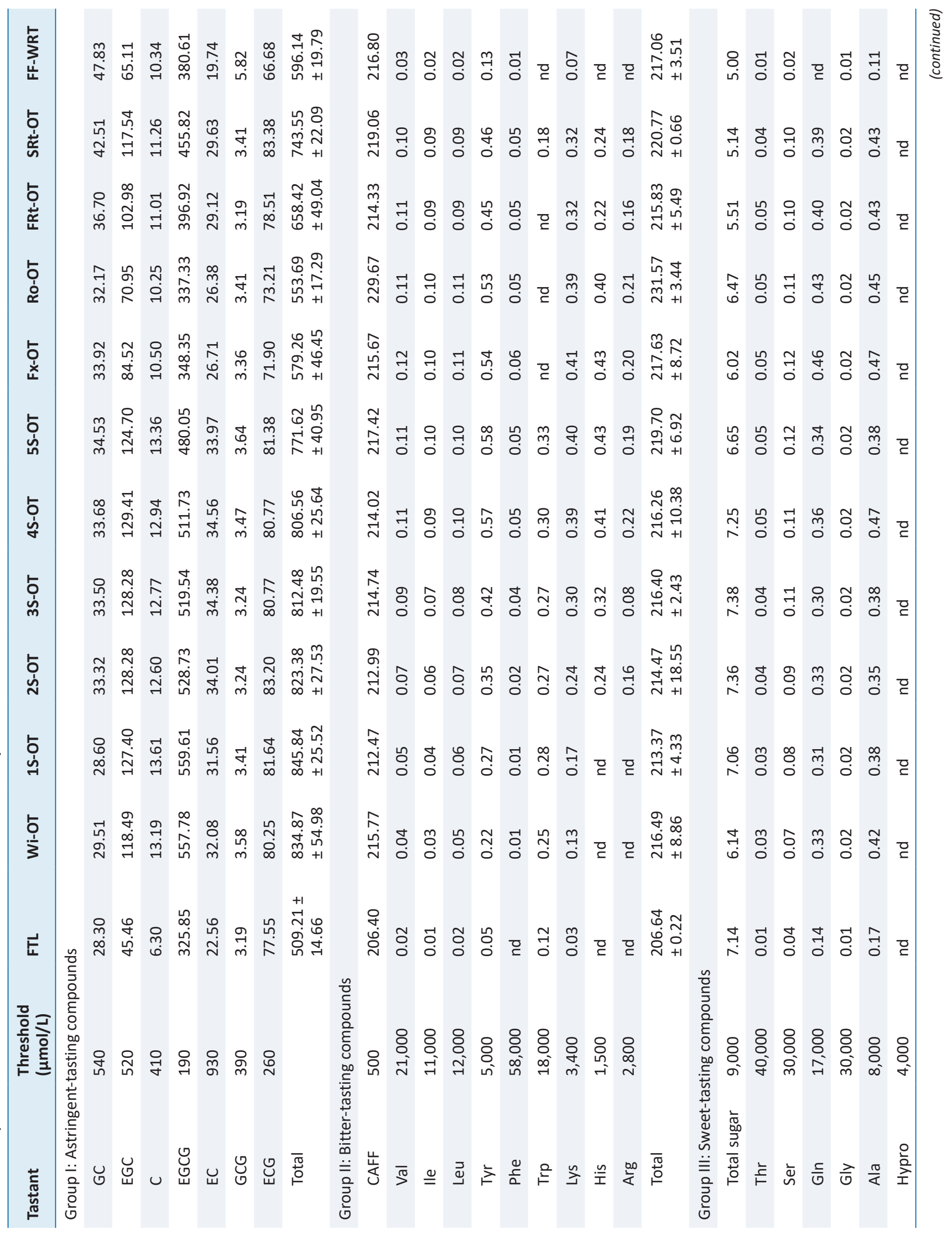




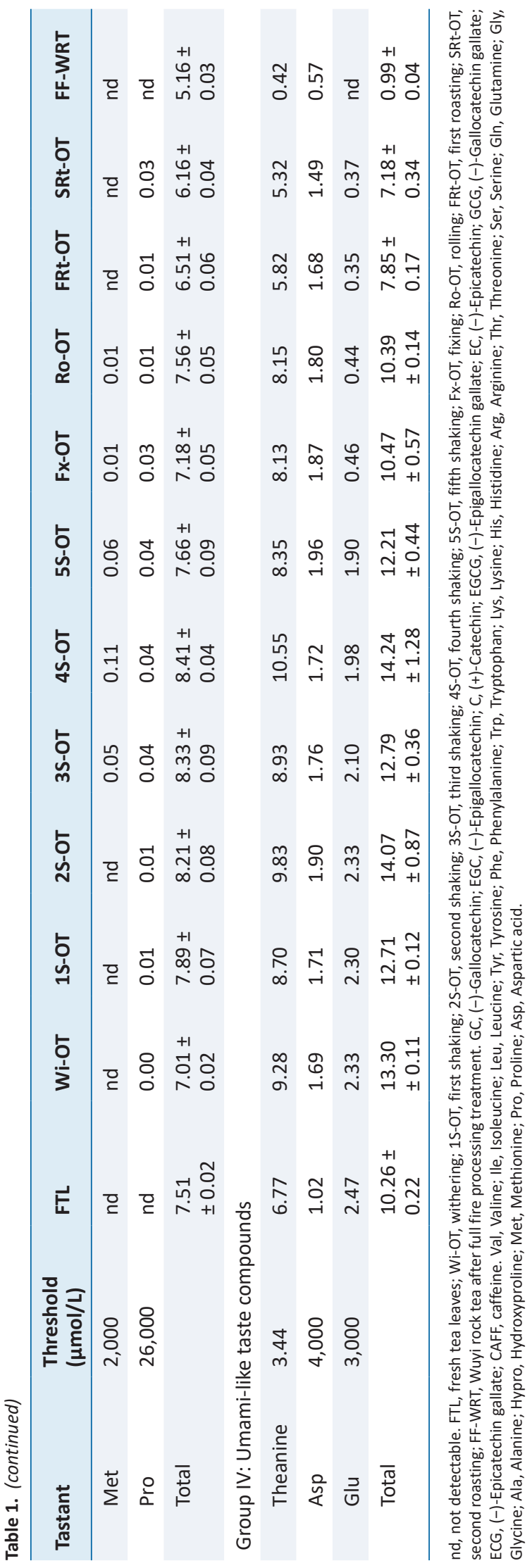

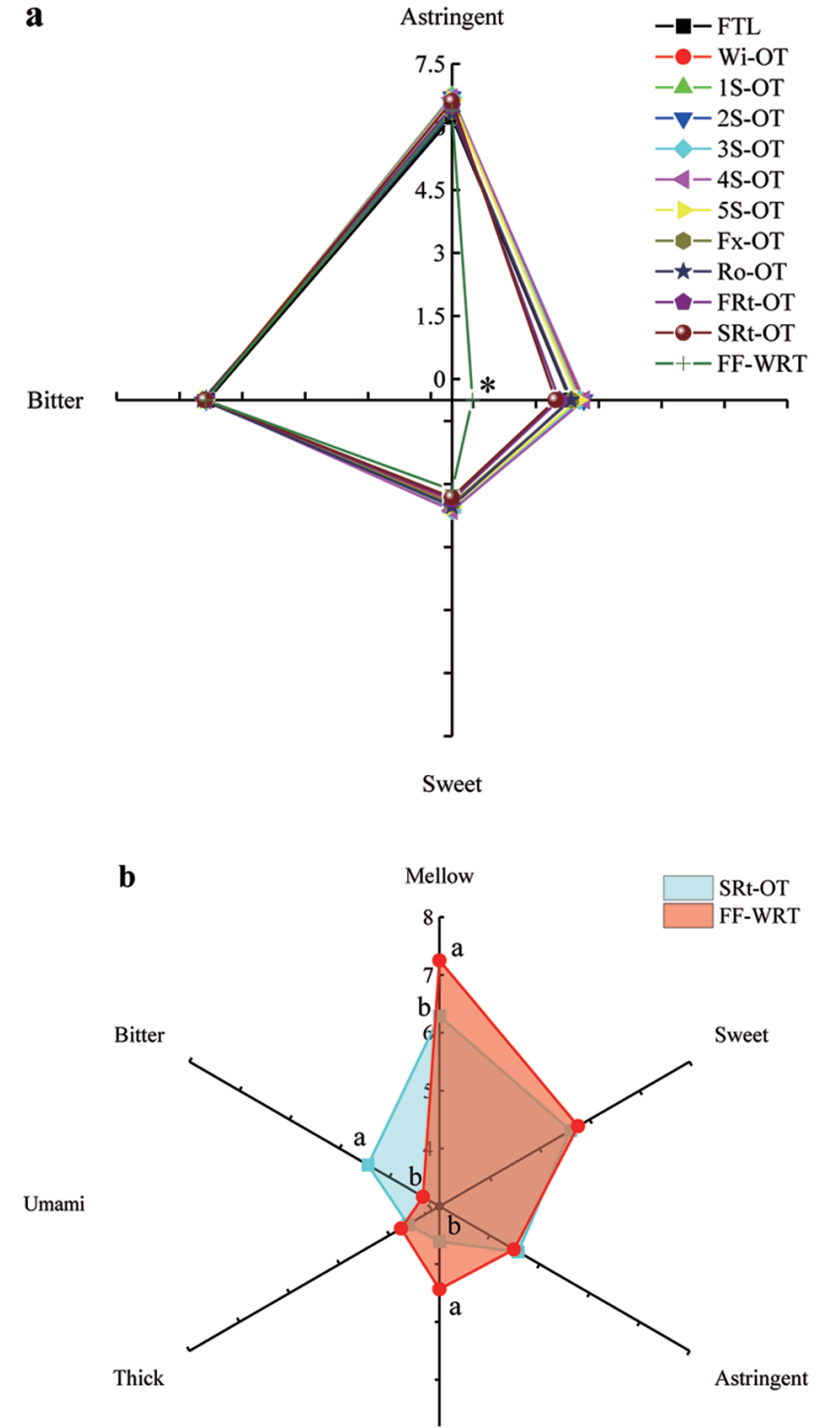

Figure 5. The taste profiles of Wuyi rock tea during manufacturing processes. (a) The tasty series of taste chemical compounds form Wuyi rock tea during manufacturing processes based on Dose-over-threshold factors with natural logarithms computation. The data from FF-WRT in 'Umami' attribute had significant difference $(p<0.05)$ from the other samples (**' labeled). (b) Radar of sensory taste attributes profile duing Wuyi rock tea manufacturing processes (SRt-OT \& FF-WRT). The taste attributes labeled with ' $a$ ', ' $b$ ' had significantly difference $(p<0.05)$ for the two samples.

an extremely strong mellow, strong sweet, moderately astringent, heavy, thick, and bitter taste (Figure 5b). The FF-WRT samples possessed a higher intensity of mellow and heavy taste, but a lower bitter taste. No significant difference was observed in both samples for the other three taste attributes. Consistent with the prediction of the taste profiles of FF-WRT, there was no umami-like taste attribute. FF-WRT showed a significantly lower bitter taste than SRt-OT, which could be covered by the taste of other ingredients (Khanum et al., 2017). The results of the sensory evaluation indicate that full fire processing is essential for the generation of the WRT taste characteristics, as it enhances the mellow, heavy taste and weakens the bitter taste, while sweetness, astringency and thick taste is maintained. 


\section{Conclusions}

Although Wuyi rock tea is appreciated for its 'rock flavor', and is gaining popularity for its special taste and aroma, there is no comprehensive comparative analysis of the effects of the individual production steps on the quality of the product. The present work indicates that tea manufacturing has an important effect on the WRT taste, which can be judged by the changes occurring in the chemical composition of caffeine, catechins, gallic acid, L-theanine, total sugar and free amino acids. In addition, taste profiles calculated by Dose-over-threshold values and sensory evaluation during the WRT manufacturing processes showed significant differences. Taste profiles were predicted by calculating the Dot factors of compounds with similar taste qualities, such as astringent, bitter, sweet and umami taste attributes during the WRT processing. Consistent with the sensory evaluation, there was no umamilike taste in FF-WRT after full fire processing, which was crucial for the final taste formation of WRT. Full fire processing enhanced the intensity of mellow, and heavy taste, while it weakened the intensity of the bitter taste. EGCG, caffeine and L-theanine were the main taste compounds in finished product tea and the processing samples with high Dot values, and were the main contributor for the astringent, bitter and umami taste, respectively. To a certain extent, the use of taste profiles can predict the basic taste impressions, but these should always be combined with a sensory evaluation in order to fully reveal the taste properties of the tea. Furthermore, the 'Rock flavor' of WRT should be the comprehensive presentation of aroma and taste, so further studies are needed to investigate the change of volatile and nonvolatile components and detail sugar profiles during tea processing and reveal the characteristic flavor profiles of mineral note and charcoal roasted odor.

\section{Acknowledgments}

We are grateful to the financially supported by the National Natural Science Foundation of China (32072634). Meanwhile, financial support was received from National Natural Science Foundation of China (22078198), Natural Science Foundation of Guangdong Province, China (2021A1515010687), and Basic Research Project of Shenzhen city, China (JCYJ20170818093429961).

\section{Conflict of interest}

The authors declare that they have no conflicts of interest to this work.

\section{Supplementary material}

Table S1. The related processing parameters of Wuyi rock tea.

Table S2. The related information of chemicals used in the research

Table S3. The detailed score on each taste attribute of WRT samples.

\section{References}

Bertuzzi, T., Martinelli, E., Mulazzi, A., and Rastelli, S. (2020). Acrylamide determination during an industrial roasting process of coffee and the influence of asparagine and low molecular weight sugars. Food Chem. 303: 125372.

Chen, Y.L., Duan, J., Jiang, Y.M., Shi, J., Peng, L., Xue, S., and Kakuda, Y. (2010). Production, quality, and biological effects of oolong Tea ( $\mathrm{Ca}-$ mellia sinensis). Food Rev. Int. 27(1): 1-15.

Cho, J.Y., Mizutani, M., Shimizu, B., Kinoshita, T., Ogura, M., Tokoro, K., Lin, M.L., and Sakata, S. (2007). Chemical profiling and gene expression profiling during the manufacturing process of Taiwan oolong tea "Oriental Beauty". Biosc. Biotechnol. Biochem. 71(6): 1476-1486.

Deng, W.W., Ogita, S., and Ashihara, H. (2009). Ethylamine content and theanine biosynthesis in different organs of Camellia sinensis seedlings. Naturforsch 64c: 387-390.

Guo, X., Ho, C.T., Schwab, W., and Wan, X. (2021). Effect of the roasting degree on flavor quality of large-leaf yellow tea. Food Chem. 347: 129016.

Guo, X., Ho, C.T., Schwab, W., Song, C., and Wan, X. (2019). Aroma compositions of large-leaf yellow tea and potential effect of theanine on volatile formation in tea. Food Chem. 280: 73-82.

Guo, X., Ho, C.T., Wan, X., Zhu, H., Liu, Q., and Wen, Z. (2021). Changes of volatile compounds and odor profiles in Wuyi rock tea during processing. Food Chem. 341: 128230.

Guo, X., Song, C., Ho, C.T., and Wan, X. (2018). Contribution of L-theanine to the formation of 2,5-dimethylpyrazine, a key roasted peanutty flavor in oolong tea during manufacturing processes. Food Chem. 263: 18-28.

Guo, Y., Zhu, C., Zhao, S., Zhang, S., Wang, W., Fu, H., Li, X., Zhou, C., Chen, L., Lin, Y., and Lai, Z. (2019). De novo transcriptome and phytochemical analyses reveal differentially expressed genes and characteristic secondary metabolites in the original oolong tea (Camellia sinensis) cultivar 'Tieguanyin' compared with cultivar 'Benshan'. BMC Genomics 20(1): 265-284.

Ho, C.T., Zheng, X., and Li, S. (2015). Tea aroma formation. Food Sci. Human Wellness 4(1): 9-27.

Hu, C.J., Li, D., Ma, Y.X., Zhang, W., Lin, C., Zheng, X.Q., Liang, Y.R., and Lu, J.L. (2018). Formation mechanism of the oolong tea characteristic aroma during bruising and withering treatment. Food Chem. 269: 202-211.

Jiang, H., Yu, F., Qin, L., Zhang, N., Cao, Q., Schwab, W., Li, D., and Song, C. (2019). Dynamic change in amino acids, catechins, alkaloids, and gallic acid in six types of tea processed from the same batch of fresh tea (Camellia sinensis L.) leaves. J. Food Comp. Anal. 77: 28-38.

Kausar, T., Akram, K., and Kwon, J.H. (2013). Comparative effects of irradiation, fumigation, and storage on the free amino acids and sugar contents of green, black and oolong teas. Radiat. Phys. Chem. 86: 96-101.

Khanum, H., Faiza, S., Sulochanamma, G., and Borse, B.B. (2017). Quality, antioxidant activity and composition of Indian black teas. J. Food Sci. Technol. 54(5): 1266-1272.

Kobayashi, A., Tachiyama, K., Kawakami, M., Yamanishi, T., Juan, I.M., and Chiu, W.T.F. (2014). Effects of solar-withering and turn over treatment during indoor-withering on the formation of pouchong tea aroma. Agric. Biol. Chem. 49(6): 1655-1660.

Kuo, P.C., Lai, Y.Y., Chen, Y.J., Yang, W.H., and Tzen, J.T. (2011). Changes in volatile compounds upon aging and drying in oolong tea production. J. Sci. Food Agric. 91(2): 293-301.

Lin, S.Y., Chen, Y.L., Lee, C.L., Cheng, C.Y., Roan, S.F., and Chen, I.Z. (2015). Monitoring volatile compound profiles and chemical compositions during the process of manufacturing semi-fermented oolong tea. J. Hortic. Sci. Biotechnol. 88(2): 159-164.

Lin, S.Y., Lo, L.C., Chen, I.Z., and Chen, P.A. (2016). Effect of shaking process on correlations between catechins and volatiles in oolong tea. J. Food Drug Anal. 24(3): 500-507.

Liu, P.P., Yin, J.F., Chen, G.S., Wang, F., and Xu, Y.Q. (2018). Flavor characteristics and chemical compositions of oolong tea processed using different semi-fermentation times. J. Food Sci. Technol. 55(3): 1185-1195.

Ma, C., Li, J., Chen, W., Wang, W., Qi, D., Pang, S., and Miao, A. (2018). Study of the aroma formation and transformation during the manufacturing process of oolong tea by solid-phase micro-extraction and gas chromatography-mass spectrometry combined with chemomet- 
rics. Food Res. Int. 108: 413-422.

Mao, A., Su, H., Fang, S., Chen, X., Ning, J., Ho, C.T., and Wan, X. (2018). Effects of roasting treatment on non-volatile compounds and taste of green tea. Int. J. Food Sci. Technol. 53(11): 2586-2594.

Nie, C., Zhong, X., He, L., Gao, Y., Zhang, X., Wang, C.M., and Du, X. (2019). Comparison of different aroma-active compounds of Sichuan Dark brick tea (Camellia sinensis) and Sichuan Fuzhuan brick tea using gas chromatography-mass spectrometry (GC-MS) and aroma descriptive profile tests. Eur. Food Res. Technol. 245(9): 1963-1979.

ÖLmez, H., and Yilmaz, A. (2009). Changes in chemical constituents and polyphenol oxidase activity of tea leaves with shoot maturity and cold storage. J. Food Process. Preserv. 34: 653-665.

Qu, F., Zhu, X., Ai, Z., Ai, Y., Qiu, F., and Ni, D. (2019). Effect of different drying methods on the sensory quality and chemical components of black tea. LWT - Food Sci. Technol. 99: 112-118.

Scharbert, S., and Hofmann, T. (2005). Molecular definition of black tea taste by means of quantitative studies, taste reconstitution, and omission experiments. J. Agric. Food Chem. 53: 5377-5384.

Seto, R., Nakamura, H., Nanjo, F., and Hara, Y. (1997). Preparation of epimers of tea catechins by heat treatment. Biosci. Biotechnol. Biochem. 61(9): 1434-1439.

Shahidi, F., Aishima, T., Abou-Gharbia, H.A., Youssef, M., and Shehata, A.A
(1997). Effect of processing on flavor precursor amino acids and volatiles of sesame paste (tehina). J. Amer. Oil Chem. Soc. 74(6): 667-678.

Sharma, E., Joshi, R., and Gulati, A. (2018). L-Theanine: an astounding sui generis integrant in tea. Food Chem. 242: 601-610.

Sheibani, E., Duncan, S.E., Kuhn, D.D., Dietrich, A.M., Newkirk, J.J., and O'Keefe, S.F. (2016). Changes in flavor volatile composition of oolong tea after panning during tea processing. Food Sci. Nutr. 4(3): 456-468.

Wang, L.F., Kim, D.M., and Lee, C.Y. (2000). Effects of heat processing and storage on flavanols and sensory qualities of green tea beverage. J. Agric. Food Chem. 48: 4227-4232.

Yi, T., Zhu, L., Peng, W.L., He, X.C., Chen, H.L., Li, J., Yu, T., Liang, Z.T., Zhao, Z.Z., and Chen, H.B. (2015). Comparison of ten major constituents in seven types of processed tea using HPLC-DAD-MS followed by principal component and hierarchical cluster analysis. LWT - Food Sci. Technol. 62(1): 194-201.

Yu, P., Yeo, A.S., Low, M.Y., and Zhou, W. (2014). Identifying key non-volatile compounds in ready-to-drink green tea and their impact on taste profile. Food Chem. 155: 9-16.

Zhang, J.N., Sun-Waterhouse, D., Su, G., and Zhao, M. (2019). New insight into umami receptor, umami/umami-enhancing peptides and their derivatives: A review. Trends Food Sci. Technol. 88: 429-438. 